\title{
Hospitalization as a trigger for venous thromboembolism - results from a population-based case-crossover study
}

Esben Bjøri*, Håkon S. Johnsen*, John-Bjarne Hansen*†, Sigrid K. Brækkan*†

*K.G. Jebsen Thrombosis Research and Expertise Center, Department of Clinical Medicine, UiT - The Arctic University of Norway, Troms $\varnothing$, Norway

†Division of Internal Medicine, University Hospital of North Norway, Troms $\varnothing$, Norway

Word count abstract: 247

Total word count: 4901

No. of references: 26

No. of tables: 4

No. of figures: 1

Abbreviated title: Hospitalization as a trigger for VTE

\section{Correspondence to:}

Esben Bjøri

K.G. Jebsen Thrombosis Research and Expertise Center (TREC)

Department of Clinical Medicine, UiT - The Arctic University of Norway

N-9037 Troms $\varnothing$, Norway

E-mail: esben.bjori@uit.no

Telephone: +4799274024 


\section{Highlights}

- Studies on the impact of hospitalization as a trigger factor for VTE are limited.

- We explored this association in a case-crossover study of cancer-free VTE patients.

- Hospitalization without concurrent immobilization was a major trigger for VTE.

- Immobilization augmented the VTE-risk substantially in hospitalized patients. 


\section{Abstract}

Background: Previous studies have reported that around $50 \%$ of patients with venous thromboembolism (VTE) has undergone recent hospitalization. However, studies on the impact of hospitalization as a trigger factor for VTE are limited.

Objectives: To investigate the impact of hospitalization with and without concurrent immobilization as a trigger factor for VTE.

Methods: We conducted a case-crossover study of 530 cancer-free VTE patients. Hospitalizations were registered during the 90-day period preceding the VTE diagnosis (hazard period), and in four preceding 90-day control periods. A 90-day washout period between the control- and hazard periods was implemented to avoid potential carry-over effects. Conditional logistic regression was used to calculate odds ratios (OR) of VTE according to hospitalization.

Results: In total, 159 (30\%) of the VTE-patients had been hospitalized in the hazard period, and the OR of hospitalization was 9.4 ( $95 \% \mathrm{Cl}: 6.8-12.8)$. The risk increased slightly with the total number of days spent in hospital (OR per day: $1.11,95 \% \mathrm{Cl}$ : 1.04-1.18), and with the number of hospitalizations (OR 8.9, 95\% Cl: 6.4-12.4 for 1 hospitalization and OR 12.3, 95\% $\mathrm{Cl}$ 6.4-23.6 for $\geq 2$ hospitalizations). Hospitalization without immobilization was 6-times (OR: 6.3, 95\% Cl: 4.4-9.2) more common, whereas hospitalization with immobilization was near 20-times (OR: 19.8, 95\% Cl: 11.5-34.0) more common in the 90-days prior to a VTE compared to the control periods.

Conclusions: Hospitalization is a major trigger factor for VTE also in the absence of immobilization. However, immobilization contributes substantially to the risk of VTE among hospitalized patients.

Keywords: Epidemiology, Hospitalization, Trigger Factors, Venous Thromboembolism (VTE) 


\section{Introduction}

Venous thromboembolism (VTE), a conceptual term for deep vein thrombosis (DVT) and pulmonary embolism (PE), is a common disease with severe complications [1-5]. Population based studies have indicated that around $40-60 \%$ of the VTE cases can be attributed to current or recent hospitalization or nursing home residency $[5,6]$. Case-control studies have reported a 7 to 21 -fold increased risk of VTE following recent hospitalization [7, 8]. Moreover, a longitudinal study from Olmsted County (US), reported that the risk of experiencing a first or recurrent VTE was 35-fold increased during the 92 days following a hospitalization [9]. Although hospitalization is acknowledged as a risk factor for VTE, the role of hospitalization as a trigger factor for VTE has not been extensively studied.

Hospitalization is often accompanied by immobilization. Immobilization is associated with a 2 to 11 -fold increased risk of VTE among hospitalized patients $[10,11]$, and up to $25 \%$ of medical patients developing a hospital-related VTE has been shown to be immobilized preceding the event $[12,13]$. Thus, the increased risk of VTE observed in hospitalized patients may be partly explained by immobilization. Most previous studies have not been able to disentangle this relationship due to lack of information on immobilization, and some studies have even used hospitalization as a proxy for immobilization [14]. The influence of immobilization on the risk of hospital-related VTE, and to what extent hospitalization without concurrent immobilization serves as a trigger of VTE, have not been well addressed.

In the present study, we set out to investigate the impact of hospitalization as a trigger of VTE, and to explore the influence of immobilization on this relationship in a population-based casecrossover study of VTE patients. We also investigated the influence of hospital-related factors, such as length of hospital-stay and frequency of hospital admissions, on the risk of VTE. Our hypothesis was that hospitalization is a major trigger for VTE also in the absence of immobilization, and that the triggering effect is influenced by the length of hospital-stay and the frequency of hospital-admissions. 


\section{Methods}

\section{Study population and outcome assessment}

The source population comprised of subjects participating in the fourth survey of the Troms $\varnothing$ study, a single-center, population based, prospective cohort study, with repeated health surveys of the inhabitants in the municipality of Troms $\varnothing$, Norway. The fourth survey was conducted in 1994/95, and included 27158 inhabitants above 24 years. Further details about the Troms $\varnothing$ study can be found elsewhere [15]. All participants gave an informed written consent, and the study was approved by The Regional Committee of Medical and Health Research Ethics. Participants were followed from the inclusion date (1994/95) through December 31, 2012, and all first-lifetime symptomatic, objectively confirmed VTE events $(n=707)$ during the course of follow-up were recorded by thorough identification and validation as previously described [16]. These 707 patients formed the basis of our case-crossover study.

\section{Study design}

In the case-crossover design, each case serves as his or her own control (self-matching), thereby controlling for risk factors that are constant within an individual (e.g. inherited thrombophilia), but vary between study objects. We defined the 90 -day period prior to the VTE as the hazard period, and 4 consecutive 90-day periods preceding the hazard period as control periods (C1-C4). A 90-day washout period was implemented between the risk and control periods, to avoid potential carry-over effects (Figure 1). This allows for comparison of exposure-frequency in the hazard period to control periods, and makes the design especially suited to study the effect of transient exposures (e.g. hospitalization) on acute events (e.g. VTE). Patients with cancer in the hazard period were excluded ( $n=177$ ), as cancer progression may change an individual's VTE risk even over a short time-period, and thereby potentially introduce confounding. Consequently, 530 cancer-free VTE patients were included in our case-crossover study.

\section{Measurements}


Trained personnel reviewed the medical records for each VTE case, and systematically collected information on potential trigger factors for each of the 90-day periods using standardized forms. Moreover, diagnostic procedures, surgical and medical treatment, laboratory tests and diagnosis during hospital admissions, day-case and outpatient clinic visits in any of the control or hazard periods were recorded. Exposures extending over several days, were registered and considered to have occurred if any of the days of exposure fell within the specified 90-day period.

Hospitalization was defined as being admitted to the hospital for more than 48 hours in the control or hazard periods. Hospital admissions >80 days were not registered as hospitalizations, as these were likely to be admitted to rehabilitation wards. The date of hospital admission and hospital discharge was used to estimate the length of hospital stay for each hospital contact. Re-admissions during each 90-day period were registered individually, and the total number of hospitalizations and total number of days spent in hospital was calculated for each 90-day period. Hospitalizations were categorized according to the main diagnosis assigned by the treating physician(s) using the $9^{\text {th }}$ and $10^{\text {th }}$ revisions of the International Classification of Diseases and Related Health Problems (ICD-9 and ICD-10). Each hospital admission was assessed individually, and patients could therefore contribute with multiple hospitalizations within each case or control period. Patients were classified in 7 broad categories, i.e. infection, chronic obstructive pulmonary disease (COPD), heart failure, acute coronary syndrome (ACS), neurologic disease, surgery (i.e. both major or minor, or admission to any surgical ward) or trauma, and others.

Immobilization was defined as the presence of one or more of the following; confinement to bed $\geq 3$ days, ECOG score of four, or other immobilizing factors specified in the patient's medical record (e.g. transient or persistent use of wheelchair, cast immobilization, etc.). CRP was analyzed in serum with a particle-enhanced immunoturbidimetric assay at the Department of Clincal Chemistry.

\section{Statistical analysis}

All statistical analysis were performed using STATA version 14.0 (Stata Corporation LP, College Station, Texas, USA). Baseline characteristics are given as means \pm 1 SD or percentages. Conditional 
logistic regression was used to calculate odds ratios (ORs) with 95\% confidence intervals (CI) for hospitalization in the hazard and control periods, as well as for the influence of duration of hospital stay and number of hospital admissions on the risk of VTE. Duration of hospital stay was analyzed as a continuous variable, and the OR was expressed per 1-day increase in hospital stay. To separate the effect of total days in hospital from frequency of admissions on the VTE risk, we performed a separate analysis adjusting the number of hospital admissions for the length of hospital stay. In order to address the impact of hospitalization as a trigger in the absence of immobilization, we performed an analysis with exposure categorized as not hospitalized, hospitalized without immobilization and hospitalized with immobilization. Since surgery is recognized as a strong trigger for VTE, we performed a separate analysis restricted to patients who did not have surgery.

Hospitalization with immobilization could possibly reflect a more severe underlying condition and a worse health condition in general. To test this hypothesis, we estimated the mean maximum CRP-level (mg/L) during hospitalization in patients with and without immobilization, as a proxy for the inflammatory state. Furthermore, to examine the potential role of confounding by occult cancer, we performed sensitivity analysis excluding patients who developed cancer in the following year after VTE.

\section{Results}

Characteristics of the 530 VTE patients are given in Table 1. The mean age was 68 years, $54.1 \%$ were women and $17.7 \%$ were obese. There were 296 (55.8\%) DVTs and 228 (43.0\%) PEs with or without concurrent DVT. Among the VTE's, 84.0\% (445) were community acquired, 10.6\% (56) acquired their VTE in-hospital, and 5.4\% (29) were nursing home residents. An overview of the categorization of hospital admissions according to the main diagnosis assigned by the treating physician in the hazard and control periods is provided in table 2. There were no substantial differences in the reason for hospitalization in the hazard compared to the control periods, except that hospitalization with heart failure was more common in the hazard period than in control periods ( $4.0 \%$ vs. $0.6 \%$ ). 
The OR according to hospitalization, length of hospital stay and number of hospitalizations, in hazard and control periods are shown in Table 2. Overall, 30.0\% $(n=159)$ of the patients had been hospitalized at least once in the hazard period $(n=530)$, compared to $6.2 \%(n=132)$ in the control periods ( $n=2120$ ). The hospital admissions were evenly distributed among the four control periods, with $5.9 \%$ ( $n=31)$ occurring in $C 1,5.5 \%(n=29)$ in $C 2,6.4 \%(n=34)$ in $C 3$ and $7.2 \%(n=38)$ in $C 4$, respectively. Multiple hospitalizations were more common in the hazard period than in the control periods (5.5\% vs. $1.6 \%)$, and patients were generally hospitalized for a longer time in the hazard period than in the control periods (median of 11 days, IQR: 6-18 vs. median of 6 days, IQR: 3-12). The OR for hospitalization as a trigger of VTE was 9.4 (95\% Cl: 6.8-12.8) (Table 2). The OR increased according to the number of hospitalizations within each period from 8.9 (95\% Cl: $6.4-12.4)$ in those with one hospitalization to $12.3(95 \% \mathrm{Cl} 6.4-23.6)$ in those with $\geq 2$ hospitalizations. After adjusting the number of hospitalization for the total number of days spent in hospital, there was no significant difference in the VTE risk between those with one compared to patients with two or more hospitalizations (OR: $1.8,95 \% \mathrm{Cl}: 0.6-5.2$ ). Overall, there was an $11 \%$ increased odds per one day increase in the total number of days spent in hospital (OR: 1.11, $95 \% \mathrm{Cl}: 1.04-1.18)$, and the OR for hospitalization $\geq 5$ days was 5.2 ( $95 \% \mathrm{Cl}$ : 1.8-15.1), compared to patients hospitalized for 1-4 days (Table 2). These results remained unchanged after adjustment for the frequency of hospital admissions (data not shown).

The ORs according to hospitalization with and without immobilization are shown in Table 3. Overall, 74 (46.5\%) of the 159 patients hospitalized in the hazard period were considered to be immobilized, compared to 34 (25.8\%) of the 132 patients hospitalized in the control periods. Hospitalization without immobilization was 6-times (OR: 6.3, 95\% Cl: 4.4-9.2) more common, whereas hospitalization with immobilization was near 20-times (OR: 19.8, 95\% Cl: 11.5-34.0) more common in the 90-days prior to a VTE compared to the control periods. The results were essentially similar when the analyses were restricted to those who did not have surgery in the hazard period, 
with an OR of $5.0(95 \% \mathrm{Cl}: 3.2-7.9)$ and $14.4(95 \% \mathrm{Cl}: 7.4-27.9)$ for hospitalization without and with immobilization, respectively (Table 3).

Immobilization during the hospital stay could reflect a more severe underlying condition, and therefore we recorded the maximum CRP levels measured during the hospital stay for each patient. The mean maximum CRP-level was $109 \pm 96 \mathrm{mg} / \mathrm{L}$ in hospitalized patients who were immobilized and $82 \pm 89 \mathrm{mg} / \mathrm{L}$ in hospitalized patients who were not immobilized. Sensitivity analysis excluding patients who developed cancer in the following year $(n=18)$ after VTE produced essentially similar results (data not shown).

\section{Discussion}

In the present case-crossover study, we found that hospitalization was a major trigger associated with a 9-fold higher risk of VTE. The triggering effect of hospitalization was mainly dependent on the length of hospital stay, but not the frequency of hospital admissions. The risk of VTE increased with $11 \%$ per one day increase in total days spent in hospital during the 90-day hazard period, and the risk was 5-fold in those with hospital admissions for $\geq 5$ days compared to those with shorter hospital stays (i.e. 1-4 days). Furthermore, we found that hospitalization without immobilization was over 6times more common, and that hospitalization with immobilization was near 20-times more common, in the 90-day period preceding a VTE compared to the control periods. The results were comparable when the analyses were restricted to patients who did not undergo surgery. Our results indicate that hospitalization is a major trigger factor for incident VTE also in the absence of immobilization. Moreover, our findings confirm that concomitant immobility increases the risk of VTE among hospitalized patients.

Several studies have investigated hospitalization as a risk factor for VTE. In a nested casecontrol study of 625 patients with a first lifetime VTE and 625 patients without VTE, hospital or nursing home confinement (institutionalization) was an independent risk factor for VTE, with an OR of 8.0 [7]. When the analysis was stratified according to institutionalization with or without recent 
surgery, the odds of VTE was almost 22-fold and 8-fold increased, respectively, compared to patients with neither institutionalization nor recent surgery. In the AT-AGE study, a case-control study of elderly individuals, hospitalization was associated with an almost 15 -fold increased risk of VTE within the first 2 weeks after hospital-discharge [8]. The risk was similar in surgery- and non-surgery-related hospitalizations (OR: 6.6 and 5.5, respectively), when compared to individuals without hospitalization. Furthermore, in a recent cohort study with hospitalization as a time-varying covariate, the risk of VTE was 35-fold increased in the period up to 92 days following a hospitalization [9].

Case-crossover studies are suitable to study triggers of acute diseases, since they are designed to answer the question "why did this disease occur right now?". This is in contrast to casecontrol and cohort studies which compare the risk between individuals, and thereby are designed to answer the question "why me?". Although previous studies have addressed hospitalization as a risk factor for VTE, few studies have investigated the role of hospitalization as a trigger of VTE using a case-crossover design. In one previous case-crossover study, any non-surgical hospitalization or skilled nursing home facility stay was found to be a significant trigger associated with a 4.2-fold higher risk of VTE [14]. Interestingly, adjustment for other hospital-related factors like major surgery, infection, blood transfusion, use of central venous catheters, injuries and medication, did not markedly influence the risk estimates, even though many of these factors most likely are in the causal pathway.

Few observational studies have evaluated the influence of length of hospitalization and frequency of hospital admissions on the risk of VTE. In a case-control study of older adults ( $\geq 60$ years) [17], Yousuf et al found that hospitalization for 4-6 days and for $\geq 7$ days was associated with a 2.4and 3.4-fold increased risk of VTE compared to patients who were hospitalized for 0-3 days. In a matched case-control study of outpatients with a VTE diagnosis during the 90-days following hospital discharge [18], increasing number of hospitalizations and increasing length of hospital stay was both associated with post-discharge VTE diagnosis. The VTE risk doubled for each additional hospital- 
admission and increased by $17 \%$ for each additional day spent in the hospital. In the present study, we found that the risk of VTE increased with $11 \%$ per one day increase in total days spent in hospital during the 90-day hazard period, and the risk was 5-times higher in those with hospital admissions $\geq 5$ days compared to 1-4 days. Conversely, we did not find any substantial differences in the VTE-risk in those with multiple hospitalizations compared to those with a single hospitalization prior to their VTE. Furthermore, after conditioning on the length of hospital stay there was no differences in the risk of VTE in those with one compared to $\geq 2$ hospitalizations, placing further emphasis on hospitalization as a high risk situation that is mainly dependent on the length of hospital stay rather than the frequency of admissions.

Immobilization is a strong trigger of VTE which often concurs with hospitalization. Few studies have been able to disentangle the effect of immobilization from that of hospitalization, and hospitalization has frequently been used as a proxy for immobilization when studying the risk of VTE, as high-quality data on immobilization is often lacking. A previous meta-analysis on immobilization and VTE-risk among hospitalized inpatients reported a relative risk of 1.5 across 7 cohort studies and an OR of 2.5 across 3 case-control studies [10]. In our study, hospitalization with immobilization was 3-times more common prior to a VTE than hospitalization without immobilization, supporting that immobilization contributes substantially to the risk of VTE among hospitalized patients. In agreement with our findings, two previous case-control studies on elderly patients, reported that immobility mediated the risk of VTE in a dose-response related manner, depending on both the type and duration of immobility [19], and that the risk was highest among patients who were bedridden in hospital $[8,19]$. Our study showed that hospitalization without immobilization was 6-times more common prior to a VTE compared to equivalent control periods. This highlights that hospitalization is a high-risk situation even in the absence of immobilization, and that thromboprophylaxis should be considered also among non-immobilized patients. The VTE risk associated with hospitalization is dependent on the reason for hospitalization (e.g. surgery, cancer, infections or acute medical conditions) [6, 20-23], as well as patient-related risk factors (e.g. age, obesity, comorbidities and 
genetic risk profile) [20-23]. Moreover, the risk can be directly related to the number of risk factors present $[20,22,24]$, as thrombosis develops once the accumulation of risk factors in an individual is sufficient to exceed the thrombosis threshold [25]. Appropriate risk stratification among hospitalized patients is therefore challenging, and further research is needed to develop risk stratification models that can accurately identify subjects at high risk of hospital-related VTE.

In addition to being a risk factor in itself, immobilization may reflect a more severe underlying disease and a generally worse health condition. Accordingly, we found a higher mean CRP-level in hospitalized patients who were immobilized compared to hospitalized patients who were not immobilized, indicating that there could be a difference in disease severity among these patients. Consequently, there could also be differences in the use of thromboprohylaxis in patients with and without restricted mobility, as immobilized patients with severe conditions are more likely to receive anticoagulant treatment. In the present study, $36.1 \%$ of the patients who were hospitalized without being immobilized, and $50.9 \%$ of those who were hospitalized with immobilization prior to their VTE received thromboprophylaxis, suggesting that our risk estimates may have been underestimated. Our data strongly indicates confounding by indication for thromboprophylaxis. Consequently, we could not adjust our analysis for thromboprophylaxis as this would introduce bias.

There are several strengths of the present study. The case-crossover design is especially suited to study triggers of disease, as information on different exposures are collected for several pre-defined time periods, allowing for comparison of exposure and exposure frequencies across different time periods in relation to the disease. Furthermore, since each subject serves as its own control, potential confounders such as chronic diseases and conditions are controlled for by the design. The present study included a large sample size of VTE patients recruited from a general population, which strengthens the external validity of our results. Moreover, the case-crossover design may partly adjust for the heterogeneity of the hospitalized population, as each subject serves as his/her own control [26]. In contrast to many other studies, we had information on immobilization 
during the hospital stay. A limitation of the case-crossover design is that it is susceptible to confounding by factors that change over time within individuals. However, this can be minimized by fitting the control periods as close to the hazard periods as possible. Moreover, all information in this study was collected retrospectively using hospital records, and the data therefore relies on thorough registration by the treating physicians and other health care professionals. Consequently, any other factors not accounted for in the medical records could potentially have influenced our results. Occult cancer could be a potential confounder in this study. However, sensitivity analysis excluding patients who developed cancer in the following year after VTE produced essentially similar results, indicating a low probability of confounding by occult cancer.

In conclusion, hospitalization is a major trigger factor for VTE also in the absence of immobilization. However, immobilization contributes substantially to the risk of VTE among hospitalized patients. Furthermore, the hospital-associated risk of VTE is mainly dependent on the length of hospital stay rather than the frequency of admissions. Our findings highlight that hospitalization is a high-risk situation also among patients who are not immobilized. 


\section{Addendum}

E. Bjøri: data analysis and writing of manuscript. H.S. Johnsen: data interpretation and revision of content. J.B. Hansen: conception and design of study, data collection and interpretation, and writing of manuscript. S.K. Brækkan: conception and design of study, data collection and interpretation, and writing of manuscript

\section{Acknowledgments}

None

\section{Sources of Funding}

K.G. Jebsen TREC is supported by an independent grant from Stiftelsen Kristian Gerhard Jebsen.

\section{Disclosures}

None.

\section{References}

1. Cushman M, Tsai AW, White RH, Heckbert SR, Rosamond WD, Enright P, Folsom AR. Deep vein thrombosis and pulmonary embolism in two cohorts: the longitudinal investigation of thromboembolism etiology. Am J Med. 2004; 117: 19-25

2. Naess IA, Christiansen SC, Romundstad P, Cannegieter SC, Rosendaal FR, Hammerstrom J. Incidence and mortality of venous thrombosis: a population-based study. J Thromb Haemost. 2007; 5: 692-9

3. Schulman S, Lindmarker P, Holmstrom M, Larfars G, Carlsson A, Nicol P, Svensson E, Ljungberg B, Viering S, Nordlander S, Leijd B, Jahed K, Hjorth M, Linder O, Beckman M. Post-thrombotic syndrome, recurrence, and death 10 years after the first episode of venous thromboembolism treated with warfarin for 6 weeks or 6 months. J Thromb Haemost. 2006; 4: 734-42

4. Piazza G, Goldhaber SZ. Chronic thromboembolic pulmonary hypertension. N Engl J Med. 2011; 364: $351-60$ 
5. Arshad N, Isaksen T, Hansen JB, Braekkan SK. Time trends in incidence rates of venous thromboembolism in a large cohort recruited from the general population. Eur J Epidemiol. 2017; 32: 299-305

6. Heit JA, O'Fallon WM, Petterson TM, Lohse CM, Silverstein MD, Mohr DN, Melton L, 3rd. Relative impact of risk factors for deep vein thrombosis and pulmonary embolism: a population-based study. Arch Intern Med. 2002; 162: 1245-8

7. Heit JA, Silverstein MD, Mohr DN, Petterson TM, O'Fallon WM, Melton L, 3rd. Risk factors for deep vein thrombosis and pulmonary embolism: a population-based case-control study. Arch Intern Med. 2000; 160: 809-15

8. Engbers MJ, Blom JW, Cushman M, Rosendaal FR, van Hylckama Vlieg A. The contribution of immobility risk factors to the incidence of venous thrombosis in an older population. $J$ Thromb Haemost. 2014; 12: 290-6

9. Heit JA, Crusan DJ, Ashrani AA, Petterson TM, Bailey KR. Effect of near-universal hospitalizationbased prophylaxis on annual number of venous thromboembolism events in the US. Blood. 2017

10. Pottier P, Hardouin JB, Lejeune S, Jolliet P, Gillet B, Planchon B. Immobilization and the risk of venous thromboembolism. A meta-analysis on epidemiological studies. Thromb Res. 2009; 124: 46876

11. Rosendaal FR. Risk factors for venous thrombotic disease. Thromb Haemost. 1999; 82: 610-9

12. Ferrari E, Baudouy M, Cerboni P, Tibi T, Guigner A, Leonetti J, Bory M, Morand P. Clinical epidemiology of venous thromboembolic disease. Results of a French Multicentre Registry. Eur Heart J. 1997; 18: 685-91

13. Barbar S, Noventa F, Rossetto V, Ferrari A, Brandolin B, Perlati M, De Bon E, Tormene D, Pagnan A, Prandoni P. A risk assessment model for the identification of hospitalized medical patients at risk for venous thromboembolism: the Padua Prediction Score. J Thromb Haemost. 2010; 8: 2450-7

14. Rogers MA, Levine DA, Blumberg N, Flanders SA, Chopra V, Langa KM. Triggers of hospitalization for venous thromboembolism. Circulation. 2012; 125: 2092-9

15. Jacobsen BK, Eggen AE, Mathiesen EB, Wilsgaard T, Njolstad I. Cohort profile: the Tromso Study. Int J Epidemiol. 2012; 41: 961-7

16. Braekkan SK, Borch KH, Mathiesen EB, Njolstad I, Wilsgaard T, Hansen JB. Body height and risk of venous thromboembolism: The Tromso Study. Am J Epidemiol. 2010; 171: 1109-15

17. Yusuf HR, Reyes N, Zhang QC, Okoroh EM, Siddiqi AE, Tsai J. Hospitalizations of adults $>/=60$ years of age with venous thromboembolism. Clin Appl Thromb Hemost. 2014; 20: 136-42

18. Herner SJ, Paulson DC, Delate T, Witt DM, Vondracek TG. Evaluation of venous thromboembolism risk following hospitalization. J Thromb Thrombolysis. 2011; 32: 32-9

19. Weill-Engerer S, Meaume S, Lahlou A, Piette F, Saint-Jean O, Sachet A, Beinis JY, Gallinari C, Grancher AS, Vincent JP, Naga H, Belmin J, Salvatore R, Kazes M, Pautas E, Boiffin A, Piera JB, Duviquet M, Knafo D, Piau A, Miric D, Jean A, Bellamy V, Tissandier O, Le Blanche AF. Risk factors for 
deep vein thrombosis in inpatients aged 65 and older: a case-control multicenter study. J Am Geriatr Soc. 2004; 52: 1299-304

20. Alikhan R, Cohen AT, Combe S, Samama MM, Desjardins L, Eldor A, Janbon C, Leizorovicz A, Olsson CG, Turpie AG, Study M. Risk factors for venous thromboembolism in hospitalized patients with acute medical illness: analysis of the MEDENOX Study. Arch Intern Med. 2004; 164: 963-8

21. Khalafallah AA, Kirkby BE, Wong S, Foong YC, Ranjan N, Luttrell J, Mathew R, Chilvers CM, Mauldon E, Sharp C, Hannan T. Venous thromboembolism in medical patients during hospitalisation and 3 months after hospitalisation: a prospective observational study. BMJ Open. 2016; 6: e012346

22. Prandoni P, Samama MM. Risk stratification and venous thromboprophylaxis in hospitalized medical and cancer patients. Br J Haematol. 2008; 141: 587-97

23. Francis CW. Clinical practice. Prophylaxis for thromboembolism in hospitalized medical patients. N Engl J Med. 2007; 356: 1438-44

24. Anderson FA, Jr., Spencer FA. Risk factors for venous thromboembolism. Circulation. 2003; 107: 19-16

25. Rosendaal FR. Venous thrombosis: a multicausal disease. Lancet. 1999; 353: 1167-73

26. Maclure M, Mittleman MA. Should we use a case-crossover design? Annu Rev Public Health. 2000; 21: 193-221 


\section{Tables}

Table 1 Characteristics of venous thromboembolism (VTE) patients ( $n=530$ )

\begin{tabular}{|c|c|c|}
\hline Age (years) & $68.4 \pm 14.0$ & \\
\hline Sex (\% women) & $54.2(287)$ & \\
\hline Obesity (\% Obese) & $17.7(94)$ & \\
\hline Deep Vein Thrombosis (\%) & $55.8(296)$ & \\
\hline Pulmonary Embolism (\%) & $43.0(228)$ & \\
\hline \multicolumn{3}{|l|}{ Location at VTE onset } \\
\hline Community & $84.0(445)$ & \\
\hline Hospital & $10.6(56)$ & \\
\hline Nursing home & $5.4(29)$ & \\
\hline \multicolumn{3}{|c|}{ Values are means \pm 1 SD or percentages with numbers in brackets. } \\
\hline & Control period & Hazard period \\
\hline & $(n=159)$ & $(n=201)$ \\
\hline Infection & $15.8 \%$ & $13.4 \%$ \\
\hline COPD* & $4.4 \%$ & $2.5 \%$ \\
\hline Heart failure & $0.6 \%$ & $4.0 \%$ \\
\hline Acute coronary syndrome & $9.5 \%$ & $6.0 \%$ \\
\hline Neurologic disease & $9.5 \%$ & $8.5 \%$ \\
\hline Surgery or trauma & $32.9 \%$ & $34.3 \%$ \\
\hline Others & $27.2 \%$ & $31.3 \%$ \\
\hline
\end{tabular}

Values are percentages with numbers in brackets.

* COPD: Chronic obstructive pulmonary disease 
Table 3 Odds ratios (ORs) of exposure in hazard period as compared to control periods

\begin{tabular}{lcccc}
\hline & $\begin{array}{c}\text { Control periods } \\
(\mathbf{n}=\mathbf{2 1 2 0})\end{array}$ & $\begin{array}{c}\text { Hazard period } \\
(\mathbf{n}=\mathbf{5 3 0})\end{array}$ & OR (95\% Cl) & ORł (95\% Cl) \\
\hline Hospitalized (n) & 132 & 159 & $9.4(6.8-12.8)$ & \\
Length of hospital stay (IQR) & $6(3-12)$ & $11(6-18)$ & $1.11(1.04-1.18)$ & \\
Hospital stay $\geq 5$ days ${ }^{*}$ & 75 & 134 & $5.2(1.8-15.1)$ & \\
Number of hospitalizations & & & & \\
0 & 1988 & 371 & Ref. & Ref. \\
1 & 109 & 130 & $8.9(6.4-12.4)$ & $1.8(0.6-5.2)$ \\
\hline 2 & 23 & 29 & $12.3(6.4-23.6)$ &
\end{tabular}

*Median (interquartile range, IQR)

tCompared to patients hospitalized for 1-4 days.

¥Adjusted for the length of hospital stay

Table 4 Odds ratios (ORs) of VTE according to hospitalization with and without immobilization.

\begin{tabular}{|c|c|c|c|c|}
\hline & $\begin{array}{l}\text { Control periods } \\
(n=2120)\end{array}$ & $\begin{array}{l}\text { Hazard period } \\
\qquad(n=530)\end{array}$ & $\begin{array}{c}\text { All } \\
\text { OR }(95 \% \mathrm{Cl})\end{array}$ & $\begin{array}{l}\text { Restricted to non-surgical* } \\
\text { OR }(95 \% \mathrm{Cl})\end{array}$ \\
\hline Not hospitalized & 1988 & 374 & Ref. & Ref. \\
\hline Hospitalized without immobilization & 98 & 85 & $6.3(4.4-9.2)$ & $5.0(3.2-7.9)$ \\
\hline Hospitalized with immobilization & 34 & 74 & $19.8(11.5-34.0)$ & $14.4(7.4-27.9)$ \\
\hline
\end{tabular}

*Analysis restricted to patients hospitalized for reasons other than surgery 


\section{Legends to figures}

Figure 1 The Case-crossover study design

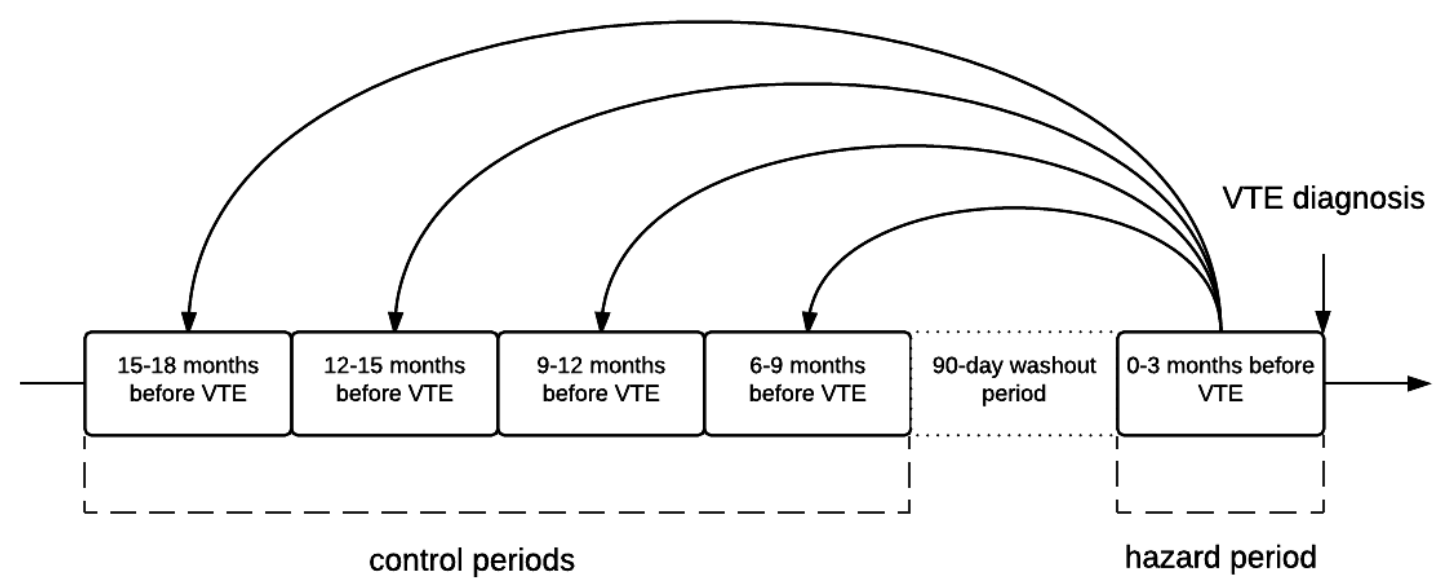

